\title{
Human Trafficking Education for Emergency Department Providers
}

\author{
Caroline Shadowen, Sarab Beaverson, and Fidelma B Rigby
}

\begin{abstract}
Many trafficked persons receive medical care in the Emergency Department (ED); however, ED staff have historically not been educated about human trafficking. In this article, we describe interventions aimed to train ED providers on the issue of trafficking. We performed a scoping review of the existing literature and found 17 studies that describe such interventions: 14 trainings implemented in the ED, two taught at conferences for ED providers, and one assessing a statemandated training. These studies demonstrate that even brief education can improve provider confidence in screening and treating patients that experienced trafficking. We advocate for interventions to promote a team-based approach specific to the ED setting, acknowledge the importance of survivors' input on curriculum development, and assess outcomes using pre- and post-surveys.
\end{abstract}

Keywords: human trafficking, education, Emergency Department, healthcare provider

Please cite this article as: C Shadowen, S Beaverson, and F B Rigby, 'Human Trafficking Education for Emergency Department Providers', Anti-Trafficking Review, issue 17, 2021, pp. 38-55, https://doi.org/10.14197/atr.201221173

\section{Introduction}

Human trafficking is a problem both worldwide and in the United States (US) ${ }^{1}$ and it can cause a variety of health issues for survivors. Trafficking survivors are at high risk of mental health issues like complex post-traumatic stress disorder (PTSD), substance use disorders, and self-harm due to repeated exposure to unpredictable physical and psychological violence. Trafficking survivors also suffer higher rates of injury, untreated chronic conditions, undesired pregnancies, and sexually

1 'Forced Labour, Modern Slavery and Human Trafficking', International Labour Organization, 2014, retrieved 18 October 2020, https://www.ilo.org/global/topics/ forced-labour/lang--en/index.htm. 
transmitted infections (STIs). ${ }^{2}$ Since trafficking relies on secrecy and isolation, it is common for trafficked persons to come into contact with professional services only when they visit a healthcare provider. ${ }^{3}$ Therefore, medical visits offer an opportunity for trafficked persons to receive much-needed medical assistance, as well as other resources for their complex psychosocial needs. ${ }^{4}$

In the US, the Emergency Department (ED) is an area of a hospital originally designated to triage and provide initial treatment for urgent or emergent medical complaints. EDs are the only care sites of the US health system mandated by federal regulation to provide care for all patients, regardless of their health condition or medical insurance coverage. ${ }^{5}$ Due to this provision, the ED has become the primary 'medical home' for many patients who have no access to other forms of healthcare. ${ }^{6}$ Over time, the ED has evolved to provide general medical care for patients with non-urgent issues (e.g., medication refill or preventative Pap smear) and to generate referrals for patients to see other providers (e.g., social workers or specialist physicians). ${ }^{7}$ The $\mathrm{ED}$ is a critical location for care provision to individuals such as trafficked persons who would otherwise have no access to medical services.

Most trafficked persons in the US interact with the medical system primarily in the ED because they lack medical insurance, regular or legal status in the country, or access to other medical care. ${ }^{8}$ Despite frequent contact with trafficked persons,

2 LJ Lederer and CA Wetzel, 'The Health Consequence of Sex Trafficking and Their Implications for Identifying Victims in Healthcare Facilities', Annals of Health Law, vol. 23, issue 1, 2014, pp. 61-91.

3 'Social Isolation and Sex Trafficking', Samuel Centre For Social Connectedness, 7 December 2015, https://www.social- connectedness.org/links-between-bullyingisolation-and-sex-trafficking.

4 L Waugh, 'Human Trafficking and the Health Care System', NCSL Legisbrief, vol. 26, issue 14, 2018, pp. 1-2.

5 WW Fields et al., "The Emergency Medical Treatment and Labor Act as a Federal Health Care Safety Net Program', Acad Emerg Med, vol. 8, issue 11, 2008, pp. 1064-1069, https://doi.org/10.1111/j.1553-2712.2001.tb01116.x.

6 K Primm, D Muraleetharan, and T Gilreath, 'Use of Emergency Departments for Preventative Care Among Adults in the United States: Estimates from the 2017 National Health Interview Survey', Public Health in Emergency Medicine, vol. 57, issue 4, 2019, pp. 578-586, https://doi.org/10.1016/j.jemermed.2019.06.004.

7 LS Zun and L Downey, 'Adult Health Screening and Referral in the Emergency Department', South Med J, vol. 99, issue 9, 2006, pp. 940-948, http://doi. org/10.1097/01.smj.0000224130.29337.19.

8 M Chisolm-Straker et al., 'Health Care and Human Trafficking: We are seeing the unseen', J Health Care Poor Underserved, vol. 27, issue 3, 2016, pp. 1220-1233, https:/ / doi.org/10.1353/hpu.2016.0131. 
ED personnel have historically not received adequate education and training about trafficking. ${ }^{9}$ Doctors, nurses, and other healthcare workers in the ED are often ill-equipped to recognise trafficked persons, screen them, or refer them to appropriate services. This lack of education and training contributes significantly to negative health outcomes for this population. ${ }^{10}$

A recent systematic review of human trafficking educational interventions demonstrated that educating healthcare providers can improve their confidence and the care they deliver to trafficked patients. ${ }^{11}$ Despite this evidence and the disproportionately high use of the ED by trafficked persons, there has been no thorough review of educational interventions on trafficking for ED providers. A review is necessary to determine how ED providers are currently educated about trafficking and where the gaps lie in training.

This article provides a scoping review of the literature on trafficking educational interventions for ED providers. We describe the interventions, summarise common themes among them, and discuss our recommendations for future educational tools, as well as describe opportunities for future research.

\section{Methodology}

We utilised the Preferred Reporting Items for Systematic Reviews and MetaAnalysis Extension for Scoping Reviews (PRISMA-ScR) ${ }^{12}$ to conduct a scoping review. We chose a scoping review because our objective was to explore the existing literature on a topic that has not been reviewed in either a scoping or systematic protocol, rather than to systematically evaluate and compare current

$9 \quad$ P Arulrajah and S Steele, 'UK Medical Education on Human Trafficking: Assessing uptake of the opportunity to shape awareness, safeguarding and referral in the curriculum', BMC Med Educ, vol. 18, issue 1, 2018, article no. 137, https://doi. org/10.1186/s12909-018-1226-y.

10 M Chisolm-Straker, LD Richardson, and T Cossio, 'Combating Slavery in the 21st Century: The role of emergency medicine', J Health Care Poor Underserved, issue 3, 2012, pp. 980-987, https://doi.org/10.1353/hpu.2012.0091.

11 HE Fraley, T Aronowitz, and HM Stoklosa, 'Systematic Review of Human Trafficking Educational Interventions for Health Care Providers', Western Journal of Nursing Research, vol. 42, issue 2, 2020, pp. 131-142, https://doi.org/10.1177/0193945919837366.

12 AC Tricco, et al., 'PRISMA Extension for Scoping Reviews (PRISMA-ScR): Checklist and explanation', Ann Intern Med, vol. 169, issue 7, 2018, pp. 467-473, https://doi. org/10.7326/M18-0850. 
literature on the topic. ${ }^{13}$

\section{Research Question}

The objective of this study was to investigate evidence addressing the research question: 'What studies exist in the current literature that aim to educate ED providers on the issue of human trafficking??

\section{Study Identification}

We searched the following databases from their inception until 2 November 2020: MEDLINE/PubMed, Ovid EMBASE, CINAHL, ERIC, MedEdPortal, Sociological Abstracts, and Academic Search Complete. The search strategies were drafted by first author Shadowen and further refined through discussion with an experienced academic librarian. We piloted the search in MEDLINE and included the following terms: ('Human Trafficking' $[\mathrm{MeSH}] \mathrm{OR}$ human trafficking OR sex trafficking) AND ('Emergency Service, Hospital'[MeSH] OR 'Emergency Medical Services'[MeSH] OR 'ED' OR 'emergency department' OR 'emergency room'). Terms were subsequently adapted to and searched in each of the included databases. A total of 757 records were identified. These references were exported into EndNote. After first author Shadowen removed duplicates, 493 unique publications remained.

As a secondary source, we searched Google Scholar from the inception of the database through 5 April 2021. First, the search included the following terms [allintitle: (education OR training) AND ("human trafficking") AND ("emergency department" OR "ED")]. Two results were identified. Next, we searched (allintitle: [education OR training] AND ["human trafficking"]). As a result of this search, 107 results were identified.

\section{Study Selection}

We used the following inclusion criteria to determine if the articles were eligible for final selection: (1) performed on human subjects; (2) related to human trafficking; (3) included an educational intervention specific to US-based ED healthcare professionals; and (4) published in English. We screened article titles

13 A systematic review is a method of research synthesis that utilises a structured protocol of retrieving and evaluating current literature to answer a research question, thereby minimising bias. A scoping review, while still considered rigorous, aims more so to evaluate gaps in knowledge in the current literature regarding a specific topic, often with the goal of acting as a precursor for a future systematic review. See Z Munn, et al., 'Systematic Review or Scoping Review? Guidance for authors when choosing between a systematic or scoping review approach', BMC Med Res Methodol, vol. 18, 2018, article 143, https://doi.org/10.1186/s12874-018-0611-x. 
and abstracts of the 602 publications that remained after our database search and excluded 557 articles because they did not meet the above criteria.

Some systematic review papers include snowballing of citations, which is when authors use a paper's citation or reference list to find additional relevant papers for the current review. ${ }^{14}$ This approach can be considered when results from a primary literature search are especially limited. Since we determined our primary and secondary searches to be adequately exhaustive, and our research team had limited resources, we chose not to perform snowballing of citations for this paper.

Authors Shadowen and Beaverson completed a full-text review for the remaining 45 articles based on the same criteria listed above. After the full-text review, 28 additional articles were excluded, and 17 articles remained.

\section{Data Extraction}

During the full-text review, the research team extracted the following information from each of the 17 included articles: citation information; article objective(s); clinical setting (e.g., urban versus rural hospital or annual patient census); description of educational intervention (e.g., time requirement or in-person versus virtual); outcomes (if measured); and unique features of each. We used a spreadsheet to track the information abstracted for each study. We decided not to assess the quality of each study using any standardised quality measure because our study was exploratory in nature and aimed to describe the existing literature on this topic and gaps therein, rather than to describe or compare the academic rigor of each study.

\section{Findings}

Overall, our scoping review yielded 17 articles describing the results of an educational intervention on human trafficking for ED providers. We summarise these studies in chronological order.

14 C Wohlin, 'Guidelines for Snowballing in Systematic Literature Studies and a Replication in Software Engineering', Proceedings of the 18th International Conference on Evaluation and Assessment in Software Engineering, Blekinge Institute of Technology, https://www.wohlin.eu/ease14.pdf. 


\section{Table 1. Summary of studies in the literature evaluating HT educational interventions for ED providers $(n=17)$.}

\begin{tabular}{|c|c|c|c|c|}
\hline Resource & Setting & Participants & $\begin{array}{l}\text { Educational } \\
\text { Intervention }\end{array}$ & Findings \\
\hline $\begin{array}{l}\text { Chisolm- } \\
\text { Straker et } \\
\text { al. (2012) }\end{array}$ & $\begin{array}{l}\text { - } 4 \text { EDs in Northeast } \\
\text { United States } \\
\text { (all with annual census } \\
>100,000 \text { ) }\end{array}$ & $\begin{array}{l}\text { - pre: } n=180 \text {, } \\
\text { intervention/ post: } \\
n=104 \\
\text { - nurses, physicians, } \\
\text { PAs, social workers, } \\
\text { medical students }\end{array}$ & $\begin{array}{l}\text { - didactic training } \\
\text { session using narrative } \\
\text { of actual encounter }\end{array}$ & $\begin{array}{l}\text { - } 98 \% \text { had never received formal } \\
\text { training on clinical presentation of } \\
\text { trafficked persons } \\
\text { - pre: } 19.2 \% \text { (very) confident in } \\
\text { identifying/ treating; post: } 90.3 \% \\
\text { - developed toolkit specific to ED } \\
\text { providers (humantraffickingED. } \\
\text { com) }\end{array}$ \\
\hline $\begin{array}{l}\text { Grace et } \\
\text { al. (2014) }\end{array}$ & $\begin{array}{l}\text { - } 20 \text { San Francisco Bay } \\
\text { Area EDs } \\
\text { - implemented in } \\
\text { division/ department } \\
\text { meetings or educational } \\
\text { Grand Rounds }\end{array}$ & $\begin{array}{l}\text { - } n=258 \\
\text { - majority ED } \\
\text { physicians } \\
\text { - physicians, nurses, } \\
\text { and social workers }\end{array}$ & $\begin{array}{l}\text { - developed w/ local } \\
\text { police }+ \text { HT educators } \\
\text { - in-person PowerPoint } \\
\text { including local cases, } \\
\text { referral options } \\
\text { - short ( } 25 \mathrm{~min}) \text { and long } \\
\text { (60min) versions }\end{array}$ & $\begin{array}{l}\text { - pre: } 7.2 \% \text { (very) knowledgeable } \\
\text { about referral resources; post: } \\
59 \% \\
\text { - pre: } 24 \% \text { knew who to call; } \\
\text { post: } 100 \% \\
\text { - no significant differences } \\
\text { between short and long versions }\end{array}$ \\
\hline $\begin{array}{l}\text { Lamb et } \\
\text { al. (2015) }\end{array}$ & $\begin{array}{l}\text { - ED in Southwestern } \\
\text { Texas } \\
\text { (annual census 173,225) } \\
\text { - at time of study, no } \\
\text { identified HT cases in ED }\end{array}$ & $\begin{array}{l}\text { - } \mathrm{n}=34 \\
\text { - attending and } \\
\text { resident physicians, } \\
\text { nurses, and medical } \\
\text { students }\end{array}$ & $\begin{array}{l}\text { - in-person, one-hour, } \\
\text { multimedia, interactive } \\
\text { presentation } \\
\text { - provided participants } \\
\text { w/ ID badge card w/ key } \\
\text { resources }\end{array}$ & $\begin{array}{l}\text { - pre: knowledgeable } 3.2 \\
\text { (on } 5 \text {-pt Likert scale); post: } 4.2 \\
\text { - pre: trauma-informed } 2.2 ; \\
\text { post: } 4.1 \\
\text { - implemented additional training } \\
\text { into continuing education }\end{array}$ \\
\hline $\begin{array}{l}\text { Cosgrove } \\
(2016)\end{array}$ & $\begin{array}{l}\text { - urban ED in South } \\
\text { Philadelphia, PA } \\
\text { (annual census } 43,000 \text { ) }\end{array}$ & $\begin{array}{l}\text { - } n=114 \\
\text { - new nurses in ED, } \\
\text { security staff, other } \\
\text { 'key personnel' }\end{array}$ & $\begin{array}{l}\text { - training from The } \\
\text { Philadelphia Anti- } \\
\text { Human Trafficking } \\
\text { Coalition (pre-tested) }\end{array}$ & $\begin{array}{l}\text { - } 100 \% \text { participants had not } \\
\text { received previous HT training } \\
\text { - pre: confidence } 1.65 \\
\text { (on } 5 \text {-pt Likert scale); post: } 4.01 \\
\text { - EMR evaluated x6mos, did not } \\
\text { identify any suspected trafficked } \\
\text { persons } \\
\text { - implemented additional training } \\
\text { into nurse triage training and } \\
\text { skills fair }\end{array}$ \\
\hline $\begin{array}{l}\text { Egyud et } \\
\text { al. (2017) }\end{array}$ & $\begin{array}{l}\text { - ED of level } 2 \text { trauma } \\
\text { center in Southwest } \\
\text { Pennsylvania } \\
\text { (annual census } 41,000 \text { ) } \\
\text { - implemented at } \\
\text { change of shift }\end{array}$ & $\begin{array}{l}\text { - } n=102 \\
\text { - nurses, physicians, } \\
\text { lab, social } \\
\text { services, radiology, } \\
\text { administration, } \\
\text { transport }\end{array}$ & $\begin{array}{l}\text { - in-person training w/ } \\
\text { supplementary video } \\
\text { materials } \\
\text { developed by } \\
\text { interprofessional team } \\
\text { - binder with materials } \\
\text { kept at nursing station }\end{array}$ & $\begin{array}{l}\text { - post-survey: } 97 \% \text { committed to } \\
\text { changing practice } \\
\text { - post: } 74 \% \text { stated education } \\
\text { improved competence } \\
\text { - EMR evaluated } \times 5 \text { mos, identified } \\
38 \text { potential victims }\end{array}$ \\
\hline $\begin{array}{l}\text { Ford } \\
\text { (2017) }\end{array}$ & $\begin{array}{l}\text { - ED in Maine hospital } \\
\text { system (annual census } \\
100,000 \text { ) } \\
\text { - implemented in } \\
\text { nursing huddle }\end{array}$ & $\begin{array}{l}\text { - } n=19 \\
\text { - nurses }\end{array}$ & $\begin{array}{l}\text { - in-person } 5 \text {-minute } \\
\text { presentation given } \\
\text { during nursing huddle }\end{array}$ & $\begin{array}{l}\text { - pre: confidence } 15 \% \\
\text { - pre: }>50 \% \text { thought it was a } \\
\text { problem in their community, } \\
\text { but }>50 \% \text { did not know proper } \\
\text { resources } \\
\text { - significant improvement in } \\
\text { knowledge questions }\end{array}$ \\
\hline $\begin{array}{l}\text { Cole et al. } \\
(2018)\end{array}$ & $\begin{array}{l}\text { - annual meeting of } \\
\text { Society for Academic } \\
\text { Emergency Medicine }\end{array}$ & $\begin{array}{l}\text { - } \mathrm{n}=19 \\
\text { - attending and } \\
\text { resident } \mathrm{ED} \\
\text { physicians }\end{array}$ & $\begin{array}{l}\text { - in-person 'train-the- } \\
\text { trainer' approach: } \\
\text { clinical scenarios + } \\
\text { teaching scripts }\end{array}$ & $\begin{array}{l}\text { - pre- and post-survey all w/ } \\
\mathrm{p}<0.001 \\
\text { - largest difference between } \\
\text { pre- and post-scores: ability to } \\
\text { instruct others }\end{array}$ \\
\hline $\begin{array}{l}\text { Normandin } \\
\text { (2018) }\end{array}$ & $\begin{array}{l}\text { - ED of urban, pediatric } \\
\text { and adult, level } 1 \\
\text { trauma center in } \\
\text { Boston, MA }\end{array}$ & $\begin{array}{l}\text { - } n=18 \\
\text { - nurses } \\
\text { - majority w/ } \\
\text { >20years ED } \\
\text { experience }\end{array}$ & $\begin{array}{l}\text { - online training } \\
\text { - included information } \\
\text { about identifying and } \\
\text { referring patients }\end{array}$ & $\begin{array}{l}\text { - no previous HT education: } 88.9 \% \\
\text { - pre score: mean } 14.06 \text {, post: } \\
28.14 \text { (significant increase, } \\
\text { p } ₫ .001 \text { ) }\end{array}$ \\
\hline $\begin{array}{l}\text { Sakamoto } \\
(2018)\end{array}$ & $\begin{array}{l}\text { - ED of women and } \\
\text { children's hospital } \\
\text { in Honolulu, Hawai'i } \\
\text { (annual census } 44,000 \text { ) } \\
\text { - implemented during } \\
\text { departmental meeting }\end{array}$ & $\begin{array}{l}\text { - pre: } n=38 \text {, } \\
\text { intervention: } n=38 \text {, } \\
\text { post: } n=48 \\
\text { - ED providers, } \\
\text { nurses, and non- } \\
\text { clinicians } \\
\text { - majority nurses } \\
\text { w/ >10years ED } \\
\text { experience }\end{array}$ & $\begin{array}{l}\text { - in-person training in } \\
\text { the ED } \\
\text { - included screening } \\
\text { questions for provider } \\
\text { use }+ \text { smart phrase } \\
\text { in EMR } \\
\text { - focus on Hawai'i data }\end{array}$ & $\begin{array}{l}\text { - pre: (somewhat) knowledgeable } \\
47.3 \% \text {; post: } 80.0 \% \\
\text { - pre: (somewhat) confident } \\
39.5 \% \text {; post: } 50.0 \% \\
\text { - EMR evaluated } x 4 \text { mos, identified } \\
2 \text { reports of suspected trafficking }\end{array}$ \\
\hline
\end{tabular}




\begin{tabular}{|c|c|c|c|c|}
\hline Resource & Setting & Participants & $\begin{array}{l}\text { Educational } \\
\text { Intervention }\end{array}$ & Findings \\
\hline $\begin{array}{l}\text { Alderman } \\
\text { (2019) }\end{array}$ & - rural ED in Virginia & $\begin{array}{l}\text { - } n=19 \\
\text { - nurses, physicians, } \\
\text { PAs, social workers, } \\
\text { NPs, nurse } \\
\text { assistants, security } \\
\text { - maj ority nurses, } \\
\text { nurse assistants }\end{array}$ & $\begin{array}{l}\text { - used HEAL Trafficking } \\
\text { and Hope for Justice } \\
\text { Protocol Toolkit (pre- } \\
\text { tested) } \\
\text { - in-person seminar }\end{array}$ & $\begin{array}{l}\text { - improvements in awareness, } \\
\text { recognition signs/sxs, } \\
\text { interviewing, intervention by } \\
\text { median } 1 \text { point on Likert scale }\end{array}$ \\
\hline $\begin{array}{l}\text { Donahue } \\
\text { et al. } \\
\text { (2019) }\end{array}$ & $\begin{array}{l}\text { - ED of community } \\
\text { hospital in suburban } \\
\text { Pennsylvania (annual } \\
\text { census } 170,000 \text { ) }\end{array}$ & $\begin{array}{l}\text { - pre survey: } n=75 \text {, } \\
\text { post: } n=56 \\
\text { - nurses, physicians, } \\
\text { NPs, PAs, } \\
\text { registration, techs } \\
\text { - maj ority nurses } \\
\text { w/ }>2 \text { years ED } \\
\text { experience } \\
\text { - }\end{array}$ & $\begin{array}{l}\text { - evidence-based } \\
\text { online training module } \\
\text { w/ PowerPoint + case } \\
\text { studies (HTEmergency. } \\
\text { com) } \\
\text { - } 20 \text { minutes }\end{array}$ & $\begin{array}{l}\text { - pre: } 89 \% \text { had not received } \\
\text { previous HT training } \\
\text { - pre: }<50 \% \text { had comprehensive } \\
\text { understanding of HT; post: } 93 \% \\
\text { - pre: confidence in treating } \\
\text { trafficked person } 4 / 10 \text {; post: } 8 / 10\end{array}$ \\
\hline $\begin{array}{l}\text { Noyes } \\
\text { (2019) }\end{array}$ & $\begin{array}{l}\text { - implemented at } \\
\text { quarterly sexual } \\
\text { assault nurse examiner } \\
\text { (SANE) training in New } \\
\text { Hampshire }\end{array}$ & $\begin{array}{l}\text { - intervention: } n=28 \text {, } \\
\text { post-survey: } n=14 \\
\text { - SANEs attending } \\
\text { training }\end{array}$ & $\begin{array}{l}\text { - in-person, } 60 \text {-minute } \\
\text { education course with } \\
\text { PowerPoint } \\
\text { - provided victim } \\
\text { identification tool } \\
\text { (validated) }\end{array}$ & $\begin{array}{l}\text { - overall agree/strongly agree } \\
\text { that information was helpful } \\
\text { - comments positive }\end{array}$ \\
\hline $\begin{array}{l}\text { Adelung } \\
(2020)\end{array}$ & $\begin{array}{l}\text { - surveyed ED } \\
\text { providers in New } \\
\text { Jersey }\end{array}$ & $\begin{array}{l}\text { - post: } n=110 \\
\text { - nurses, physicians, } \\
\text { PAs, NPs } \\
\text { - maj ority nurses w/ } \\
\text { >10yr ED experience }\end{array}$ & $\begin{array}{l}\text { - mandated training } \\
\text { (varied by location) w/ } \\
\text { average time of } 1.85 \mathrm{hrs} \\
\text { - PROTECT survey } \\
\text { instrument (pre-tested) } \\
\text { modified for the study }\end{array}$ & $\begin{array}{l}\text { - generally, perception of training } \\
\text { impact was positive } \\
\text { - } 80 \% \text { training increased HT } \\
\text { awareness 'some' to 'a lot' } \\
\text { - less of an impact on their ability } \\
\text { to treat trafficked patients }\end{array}$ \\
\hline $\begin{array}{l}\text { Cummings } \\
(2020)\end{array}$ & $\begin{array}{l}\text { - ED of small } \\
\text { community hospital in } \\
\text { Pennsylvania }\end{array}$ & $\begin{array}{l}\text { - pre: } n=38, \text { pre }+ \\
\text { intervention: } n=26 \text {, } \\
\text { post or all } 3: n=17 \\
\text { - nurses }\end{array}$ & $\begin{array}{l}\text { - education included } \\
\text { validated screening tool } \\
\text { - survey designed based } \\
\text { on evidence in literature }\end{array}$ & $\begin{array}{l}\text { - no statistically significant } \\
\text { differences between pre- and } \\
\text { post-tests } \\
\text { - postulated possibly due to } \\
\text { previous knowledge }\end{array}$ \\
\hline $\begin{array}{l}\text { Derr } \\
(2020)\end{array}$ & $\begin{array}{l}\text { - ED of large private } \\
\text { hospital in SoCal }\end{array}$ & $\begin{array}{l}\text { - pre: } n=139, \text { post: } \\
n=129 \\
\text { - all ED staff } \\
\text { including } \\
\text { environmental } \\
\text { services (EVS) }\end{array}$ & $\begin{array}{l}\text { - validated training } \\
\text { algorithm including } \\
\text { resources, red flags, } \\
\text { reporting } \\
\text { - provided signs with } \\
\text { pull tabs throughout the } \\
\text { department } \\
\text { - training to EVS in } \\
\text { English and Spanish }\end{array}$ & $\begin{array}{l}\text { - yes/no questions: pre } \\
\text { confidence to report } 83 \text {; post } 110 \\
\text { - pre knowledge } 48 \text {; post } 112 \\
\text { - EMR evaluated } \times 12 \text { mos: } 41 \\
\text { possible physical abuse, } 38 \\
\text { possible sexual abuse, } 19 \text { possible } \\
\text { exploitation }\end{array}$ \\
\hline $\begin{array}{l}\text { Tiller et } \\
\text { al. }(2020)\end{array}$ & $\begin{array}{l}\text { - ED of academic, } \\
\text { urban county hospital } \\
\text { in North Carolina } \\
\text { (annual census } 85,000 \text { ) }\end{array}$ & $\begin{array}{l}\text { - faculty, learners, } \\
\text { ancillary staff }\end{array}$ & $\begin{array}{l}\text { - used HEAL Trafficking } \\
\text { and Hope for Justice } \\
\text { Protocol Toolkit* } \\
\text { (pre-tested) } \\
\text { - in-person lecture given } \\
\text { by law enforcement }\end{array}$ & $\begin{array}{l}\text { - no surveys } \\
\text { - emphasised importance of } \\
\text { teamwork and how to give } \\
\text { resources to patients who decline } \\
\text { assistance }\end{array}$ \\
\hline $\begin{array}{l}\text { Greiner- } \\
\text { Weinstein } \\
\text { et al. } \\
\text { (2021) }\end{array}$ & $\begin{array}{l}\text { - ED of urban academic } \\
\text { hospital in Chicago } \\
\text { (annual patient census } \\
24,000)\end{array}$ & $\begin{array}{l}\text { - nurses + social } \\
\text { workers }\end{array}$ & $\begin{array}{l}\text { - e-learning educational } \\
\text { tool focusing on red } \\
\text { flags - } \\
\text { validated pre/ post-test } \\
\text { - included EMR protocol }\end{array}$ & $\begin{array}{l}\text { - exam scores: pre } 77 \% \text {; post } 93 \% \\
\text { - } 89 \% \text { would apply to practice }\end{array}$ \\
\hline
\end{tabular}

* C Ross et al., 'Human Trafficking and Health: A cross-sectional survey of NHS professionals' contact with victims of human trafficking', BMJ Open, vol. 5, issue 8, e008682, 2015, https://doi.org/10.1136/bmjopen-2015-008682.

** SB Baldwin, J Barrows, and H Stoklosa, 'Protocol Toolkit for Developing a Response to Victims of Human Trafficking', HEAL Trafficking and Hope for Justice, 2017, https://healtrafficking.org/protocol-toolkit-for-developing-a-response-to-victims-ofhuman-trafficking-in-health-care-settings. 
Generally, pre-intervention surveys indicated a lack of knowledge and confidence amongst all levels of ED staff, regardless of hospital location or the type of participants included (e.g., clinical versus non-clinical). Interventions were completed both online and in-person and most typically took place during shift changes or at department-wide meetings. Post-intervention surveys consistently demonstrated significant improvement in providers' understanding of the issue of trafficking and their confidence interacting with trafficked patients.

The settings of studies reflect the diversity of the US healthcare system, with studies taking place all over the country, including many different categories of ED staff, and in a wide variety of EDs. Interventions were implemented in locations across the US, including California, ${ }^{15}$ New Hampshire, ${ }^{16}$ and Hawai' $1 .{ }^{17}$ Most interventions were implemented in vivo in their respective ED, each of which served an annual patient census ranging from 24,000 to 173,000.

The total number of participants included in each intervention ranged from 14 (Noyes) to 258 (Grace et al.). Most of the studies included multiple categories of ED staff, while a few focused on specific groups: nurses, ${ }^{18}$ sexual assault nurse examiners or SANES (Noyes); or physicians (Cole et al.). Participants in onethird of the interventions ${ }^{19}$ involved non-clinical staff: registration and security

15 AM Grace et al., 'Educating Health Care Professionals on Human Trafficking', Pediatr Emerg Care, vol. 30, issue 12, 2014, pp. 856-861, https://doi.org/10.1097/ PEC.0000000000000287 and L Derr, 'Human Trafficking 2.0: Awareness, assessment $\&$ assistance beyond the emergency care center', St. Joseph Hospital, Orange, CA. Evidence Based Practice Conference, 26, 2020.

CL Noyes, 'Human Trafficking Recognition and Response Training for Sexual Assault Nurse Examiners in New Hampshire Emergency Departments', Doctor of Nursing Practice (DNP) Projects, 182, 2019.

17 KK Sakamoto, 'Sex Trafficking Assessment Protocol for Medical Professionals in the Emergency Department', University of Hawai'i at Manoa DNP Thesis, 2018.

18 KA Cummings, 'An Educational Intervention to Increase Emergency Department Nurse's Knowledge and Confidence in Recognizing Victims of Human Trafficking', Regis University Student Publications, thesis 982, 2020; PA Normandin, 'Human Trafficking Online Educational Training to Increase Emergency Nurses' Knowledge, Self-Efficacy, Skills in Identification, and Referral of Human Trafficking Survivors', Tufts Medical Center \& Floating Hospital for Children, 2018; and Ford.

19 C Cosgrove, 'Increasing Awareness about Human Trafficking: Education for frontline healthcare providers', Master of Public Health Thesis and Capstone Presentation, Thomas Jefferson University, presentation 198, 2016; SL Alderman, 'An Education Implementation Project in an Emergency Department to Increase Awareness and Referral for Trafficked Person Encounters', Doctoral Dissertations and Projects, Liberty University, project 2002, 2019; S Donahue, M Schwien, and D LaVallee, 'Educating Emergency Department Staff on the Identification and Treatment of Human Trafficking Victims', J Emerg Nurs, vol. 45, issue 1, 2019, pp. 16-23, https:// doi.org/10.1016/j.jen.2018.03.021; as well as Egyud et al., Sakamoto, Derr, and Tiller et al. 
personnel, patient transporters, and environmental service workers. Only a few studies $^{20}$ involved learners, such as medical students, in their interventions. A unique feature of one study (Derr) was that it offered participants the choice between an English and a Spanish language version of the intervention.

Methods for delivering the educational material similarly reflected the dynamic nature of the ED setting: two studies (Grace et al. and Sakamoto) implemented their brief training at departmental meetings or didactic sessions, and two others were delivered at shift change meetings — one multidisciplinary ${ }^{21}$ and one nursing huddle. ${ }^{22}$ Two of the interventions (Noyes and Cole $e t a .^{23}$ ) were implemented at conferences for ED providers, and one consisted of a survey emailed to professional listservs of ED providers to assess the impact of a state-mandated human trafficking training. ${ }^{24}$ Several of the studies mentioned a lack of awareness in their particular EDs on the issue of trafficking despite high numbers of trafficking cases reported locally. ${ }^{25}$

Intervention design also varied widely, with some trainings consisting of a few online modules ${ }^{26}$ and others carried out in-person (Grace et al., Lamb et al., Egyud

20 J Tiller and S Reynolds, 'Human Trafficking in the Emergency Department: Improving our response to a vulnerable population', West J Emerg Med, vol. 21, issue 3, 2020, pp. 549-554, https://doi.org/10.5811/westjem.2020.1.41690; as well as Chisolm-Straker et al., 2012, Lamb et al.

21 A Egyud, et al., 'Implementation of Human Trafficking Education and Treatment Algorithm in the Emergency Department', J Emerg Nurs, vol. 43, issue 6, 2017, pp. 526-531, https://doi.org/10.1016/j.jen.2017.01.008.

22 S Ford, 'Sexual Trafficking: Developing a teaching strategy for emergency department registered nurses', Honors College, 2017, https:/ / digitalcommons.library.umaine.edu/ honors/299.

23 MA Cole, et al., 'A Theory-based Didactic Offering Physicians a Method for Learning and Teaching Others About Human Trafficking', AEM Educ Train, vol. 2, issue s1, 2018, pp. S25-S30, https://doi.org/10.1002/aet2.10206.

24 M Adelung, 'Human Trafficking: The efficacy of a mandatory training on the competency of emergency department providers', Sigma's VIRTUAL $31^{\text {st }}$ International Nursing Research Congress, 2020, https://sigma.nursingrepository.org/bitstream/ handle/10755/21211/Adelung_Abstract.pdf.

25 GS Lamb and C Tsai, 'Human Trafficking Educational Initiative in a Pediatric Emergency Department: A multidisciplinary approach', American Academy of Pediatrics National Conference \& Exhibition, paper 32238, 2015, https:/ / aap.confex. com/aap/2015/webprogrampress/Paper32238.html.

26 G Greiner-Weinstein, A Klopp, and V Bacidore, 'Implementing an Interprofessional Human Trafficking Program in the Emergency Department: An evidence-based quality improvement initiative', Loyola Palmer Research Symposium, Asynchronous Poster \#7, 2021, https://ecommons.luc.edu/loyola_palmerresearchsymposium/2021/ Asynchronous_Posters; as well as Donahue et al. and Normandin. 
et al., Ford, Cole et al., Sakamoto, Alderman, Noyes, and Tiller et al.). In general, trainings covered how to recognise signs of trafficking, local resources, and other elements like advice from law enforcement officers and tips from social workers in the community. Four studies contained narratives of actual encounters between providers and patients (Chisolm-Straker et al., 2012, Grace et al., Cole et al., and Donahue $e t$ al.), with one of those including de-identified information from cases that had taken place locally (Grace et al.). The time required to complete the interventions ranged from 5 (Ford) to 111 minutes (Adelung). One unique study (Grace et al.) provided both a short (25-minute) and a long (60-minute) version of their educational tool. Survey instruments and trainings were all evidence-based, with three containing a validated component (Noyes, Derr, Greiner-Weinstein et al.), and four containing a pretested component (Cosgrove, Alderman, Adelung, and Tiller et al.)

Interventions were shown to be quite effective overall in improving providerreported knowledge and confidence. All studies but one (Tiller et al.) utilised pre- or post-surveys or tests to evaluate outcomes; most utilised both pre- and post-surveys, and three assessed post-survey responses only (Egyud et al., Noyes, and Adelung).

Pre-tests demonstrated a general lack of previous training on trafficking, with up to 98 per cent (Chisolm-Straker et al., 2012) and 100 per cent (Cosgrove) of participants reporting they had never received formal training on the topic. Pretest confidence was quite low amongst most participants: 15 per cent of Ford's nursing cohort indicated confidence identifying trafficked persons, and Cosgrove's participants rated their confidence a mean of 1.65 on a 5-point Likert scale, with 5 being highest confidence.

All but one of the studies (Cummings) that assessed post-survey knowledge or confidence found significant improvement post-intervention. For example, Donahue's pre-test indicated that less than 50 per cent of participants had a comprehensive understanding of trafficking, while post-test results increased to 93 per cent. In that same study, overall confidence in treating trafficked persons was 4/10 pre-test and increased to 8/10 post-test. By contrast, Cummings postulated that the lack of significant improvement post-intervention among its participants could have been due to high levels of knowledge prior to the intervention.

In addition to assessing outcomes of an intervention on providers, several studies evaluated the Electronic Medical Record (EMR) to investigate impacts on patients. Derr evaluated the EMR for 12 months after the intervention, finding nearly 100 cases of possible physical abuse, sexual abuse, or exploitation. Cosgrove evaluated the EMR for six months post-intervention and did not identify any suspected trafficked persons. Egyud evaluated the EMR for five months post-intervention and identified 38 potential victims. Sakamoto assessed the EMR for four months and found two suspected trafficked persons. 
Several studies included an extra component to aid staff in making necessary changes to their clinical care for suspected trafficked patients. Lamb provided participants with a card to attach to their ID badge with key local resources to share with patients. Sakamoto's and Greiner-Weinstein's clinical participants were given a smartphrase (an abbreviation that inserts a pre-programmed paragraph of written content into the note) or recording protocol to help with patient documentation in the EMR. The SANE participants in Noyes' study were provided with a validated victim identification tool to use when screening patients after their training. The hospital where Derr's study took place was provided with posters to hang in the ED that included local resources written on pull tabs for patients to take as needed.

\section{Discussion}

Our literature review demonstrated that all but one study assessed outcomes of a human trafficking educational intervention, demonstrating that training increased provider comfort with recognising and treating trafficked persons. Furthermore, several studies investigated patient outcomes, illuminating increased identification of and resource provision for patients post-intervention. Though there is a dearth of studies describing and evaluating human trafficking trainings for ED providers, even brief educational initiatives - especially those tailored to the unique physical and logistical constraints of an ED and informed by survivors-can improve awareness of and clinical competence in this critical area. Trafficking education has historically not been integrated into training for ED personnel and, consequently, staff have had limited confidence in their ability to address the issue. There is no evidence to our knowledge of any of the reviewed approaches being superior to the others, and comparative studies have not yet been conducted. Further research is needed to compare and validate these ED-specific educational interventions to close gaps in the current literature.

Though we found an overall lack of peer-reviewed studies on the topic of human trafficking education for ED providers, there has been increased awareness in recent years, with studies emerging in the 'grey literature' in the form of poster presentations and theses. These data support the ED as a critical place of opportunity to engage with trafficked persons in care, as well as the notion that when ED providers feel knowledgeable about how to triage and treat trafficked persons, these patients' medical care improves. ${ }^{27}$ Because the ED is often a primary 'medical home' for many vulnerable patients, it is increasingly viewed as a place

27 CL Hua, AR Bardo, and JS Brown, 'Mistrust in Physicians Does Not Explain Blackwhite Disparities in Primary Care and Emergency Department Utilization: The importance of socialization during the Jim Crow era', J Natl Med Assoc, vol. 110, issue 6, 2018, pp. 540-546, https://doi.org/10.1016/j.jnma.2018.01.006. 
where providers should receive training to give comprehensive, non-judgmental care. ${ }^{28}$ When patients feel that they are in a safe and non-biased environment, they may be more comfortable to speak about their situation and ask for resources. ${ }^{29}$ While the goal of treatment of trafficked patients is not to force disclosure, it is imperative for ED providers to create an atmosphere of support and expertise to optimise patient interactions. Comprehensive training can increase confidence and the ability to produce a safer setting.

\section{Mandated Training on Human Trafficking}

All of the studies we reviewed that assessed provider-reported measures found deficiencies prior to the educational intervention. Lack of knowledge and confidence are major barriers to optimal care provision for trafficked persons. As attention is drawn to the issue, more resources are being allocated to develop triage protocols and care partnerships ${ }^{30}$ For example, many US states now require training on human trafficking when providers recertify their licenses. ${ }^{31}$ One of the studies we reviewed (Adelung) found that mandated training was efficacious for participants because it increased baseline awareness of trafficking across different training levels. Because literature on other healthcare issues reveals that increased provider confidence can improve patient outcomes, ${ }^{32}$ our hope is that increased provider confidence in interacting with trafficked patients may improve those outcomes as well. ED administrators and providers need to be educated on trafficking as an important issue so that resources and protocols can be in place for the proper screening and treatment of patients. For example, an ED can have universal screening questions in place that ED providers ask all patients. ED providers may also be given an algorithm to follow if a patient screens positive, such as alerting a social worker and using a template to document their findings in the EMR. Furthermore, EDs can consider incorporating training materials at department meetings or educational conferences.

28 K Ruben, K Mortensen, and B Eldridge, 'Emergency Department Referral Process and Subsequent Use of Safety-Net Clinics', J Immigr Minor Health, vol. 17, issue 5, 2015, pp. 1298-304, https://doi.org/10.1007/s10903-014-0111-y.

29 RM Hermann, E Long, and RL Trotta, 'Improving Patients' Experiences Communicating with Nurses and Providers in the Emergency Department', J Emerg Nurs, vol. 45, issue 5, 2019, pp. 523-530, https://doi.org/10.1016/j.jen.2018.12.001.

30 Tiller and Reynolds.

31 'Michigan Human Trafficking: Michigan Department of Licensing and Regulatory Affairs required mandatory human trafficking training for health professionals', Biologix Solutions, retrieved 30 March 2021, https://blxtraining.com/humantrafficking-training; 'Human Trafficking', FL HealthSource, https://www. flhealthsource.gov/humantrafficking, retrieved 11 July 2021.

32 K Owens and S Keller, 'Exploring Workforce Confidence and Patient Experiences: A quantitative analysis', Patient Experience Journal, vol. 5, issue 1, 2018, pp. 97-105. 


\section{Focusing Interventions on the ED}

In addition to evaluating the impact of an educational intervention, one of the studies we reviewed aimed to develop a human trafficking screening tool specific to the needs of an ED (Chisolm-Straker et al., 2012). Despite the high prevalence of trafficking and its interface in the ED, there are few standardised screening protocols for how ED providers should treat potentially trafficked patients. When surveyed, survivors of trafficking noted that they repeatedly visited the ED and wondered why the frequency and nature of their visits did not 'raise a red flag'. ${ }^{33}$ This finding indicates the potential missed opportunities that occur for providers to connect with patients and screen them for trafficking.

For EDs that do have screening protocols, the most common assessment method are universal questions on feelings of safety (e.g., asking every patient, 'Do you feel safe at home'?). ${ }^{34}$ Many healthcare institutions-EDs in particular-have attempted to develop screening protocols to identify and treat trafficked patients. ${ }^{35}$ Methods for quick, universal screenings in the ED are currently in process of validation, ${ }^{36}$ which is greatly needed for effective care. We know from the ED literature that screening tools in the ED are most effective when they are short, computer-based, and given to patients while waiting to see a provider. ${ }^{37}$ These same characteristics should be applied to screening measures focused on potentially trafficked persons.

Several of the studies we reviewed gave nurses and physicians specific 'red flag' medical complaints to be aware of when attempting to identify trafficked persons. In the ED, trafficked persons often present themselves with acute physical manifestations of physical and sexual violence (e.g., pelvic pain, STIs, or injuries).

33 'A Roadmap for Systems and Industries to Prevent and Disrupt Human Trafficking Health Care', Polaris, retrieved 6 October 2020, https://polarisproject.org/ wp-content/uploads/2018/08/A-Roadmap-for-Systems-and-Industries-toPrevent-and-Disrupt-Human-Trafficking-Health-Care.

34 JD Dols, et al., 'Human Trafficking Victim Identification, Assessment, and Intervention Strategies in South Texas Emergency Departments', J Emerg Nurs, vol. 45, issue 6, 2019, pp. 622-633, https://doi.org/10.1016/j.jen.2019.07.002.

35 VJ Greenbaum, et al., 'Multi-level Prevention of Human Trafficking: The role of health care professionals', Prev Med, vol. 114, 2018, pp. 164-167, https://doi.org/10.1016/j. ypmed.2018.07.006.

36 M Chisolm-Straker et al., 'Building RAFT: Trafficking screening tool derivation and validation methods', Acad Emerg Med, vol. 27, issue 4, 2020, pp. 297-304, https://doi. org/10.1111/acem.13888.

37 SG Weiner, JA Hoppe, and MD Finkelman, 'Techniques to Shorten a Screening Tool for Emergency Department Patients', West J Emerg Med, vol. 20, issue 5, 2019, pp. 804-809, https://doi.org/10.5811/westjem.2019.7.42938. 
Their acute physical complaints may also manifest as traumas from hazardous work or living conditions (e.g., weight loss or dehydration).$^{38}$ Even when trafficked persons do not arrive at the ED with a life-threatening physical symptom, they need to be thoroughly examined and screened. Trafficked persons have been shown to have higher rates of chronic diseases, which may present themselves in more insidious and nuanced ways than acute conditions. ${ }^{39}$ Additional research is needed to evaluate the incorporation of trafficking-informed care into primary care, where these chronic diseases can be more thoroughly addressed and treated.

\section{Strengths of Survivor Input on Training}

The content of trafficking training is critical to its usefulness in the clinical setting. Historically, there have been multiple misrepresentations and misguided principles perpetuated in trafficking education; for example, trainings have at times overemphasised trafficking in the sex trade and under-emphasised trafficking in other sectors, ${ }^{40}$ both of which may narrow the scope of 'who' is anticipated to be a potential victim. Similarly, instructing health care providers to only focus on 'red flags', though helpful in some cases, may rely on the experience (and biases) of the providers ${ }^{41}$ and therefore impact who is screened or treated. Training, therefore, must involve a nuanced conversation about different forms of trafficking in the context of the respective local areas, including a frank dialogue about the appropriate involvement of law enforcement.

Human trafficking training for healthcare providers must be informed by survivors. One of the studies discussed in this review included what the authors referred to as a 'rescue plan' in the resources given to providers (Egyud et al.). Though treatment plans have historically stated goals like patient 'rescue' and 'disclosure', trafficking advocates called for a move away from such terminology and aims, toward a trauma-informed approach focused not on 'rescue' but rather on universal education and violence prevention that acknowledges that all patients

38 'Labor Trafficking in the US: A closer look at temporary work visas', Polaris, 2015, retrieved 8 October 2020, https://polarisproject.org/resources/labor-trafficking-inthe-us-a-closer-look-at-temporary-work-visas.

39 SJ McConkey, et al., 'Sex Trafficking in Ireland from a Health Care Perspective', Ir Med J, vol. 107, issue 9, 2014, pp. 270-272.

40 T Bonilla and $\mathrm{CH}$ Mo, 'The Evolution of Human Trafficking Messaging in the United States and Its Effects on Public Opinion', Journal of Public Policy, vol. 39, issue 2, 2019, pp. 201-234, https://doi.org/10.1017/S0143814X18000107.

41 Q Capers IV, DA Bond, and US Nori, 'Bias and Racism Teaching Rounds at an Academic Medical Center', Chest Journal, vol. 158, issue 6, 2020, pp. 2688-2694, https:// doi.org/10.1016/j.chest.2020.08.2073. 
may benefit from some form of safety contingency planning. ${ }^{42}$ We know from the literature that survivors benefit when given the opportunity to lead training development and that training is more effective with their input. ${ }^{43}$ When survivors take part in developing curricula, results are consistently culturally aware, victim and survivor-centred, and trauma-informed, which creates a more robust training for providers. ${ }^{44}$ Trafficking survivors, advocates, and community stakeholders should participate in developing educational measures to ensure appropriate and consistent goals, protocol, and language.

Several of the studies highlighted that many providers may be uncomfortable with the topic of trafficking, and trafficked persons often experience forms of trauma that providers are unfamiliar with. We advocate for providers to be reassured that their discomfort is understandable, yet it need not interfere with the care they provide. Administrators should have the resources in place to support practitioners. For example, providers who interact with a suspected trafficked patient may need to see a counsellor to discuss their experience, and they should be excused from work duties in order to do so. Further, administrators should encourage partnerships between providers and forensic nurses, social workers, and other specialists to provide trauma-informed, patient-centred care. A way to encourage such collaboration is to host trainings and discussions (traffickingspecific and otherwise) with providers from different disciplines.

\section{Importance of a Team-Based Approach}

Most studies we reviewed emphasised the team approach to emergency care in the ED, highlighting the distribution of educational interventions comprised of a variety of staff including physicians, technicians, and even non-clinical employees. We value this 'team-based' model and extend it to providers beyond the ED, because ED nurses, physicians, and social workers cannot be responsible for addressing all aspects of trafficked persons' health and social circumstances. Rather, this kind of comprehensive treatment requires a longterm multidisciplinary team. ED providers should focus on addressing urgent or emergent needs, and ED-specific training measures must equip them to do so.

42 'Lecture: Screening to identify trafficking survivors', HHS Health and Trafficking Symposium, 28 November 2018, https://www.acf.hhs.gov/sites/default/files/otip/ symposium_panel_3_screening_to_identify_trafficking_survivors.pdf.

43 R Lloyd, 'From Victim to Survivor, from Survivor to Leader', Girls Educational and Mentoring Services, https://issuu.com/gems/docs/from_victim_to_survivor_ from_survivor_to_leader.

44 H Stoklosa, A Grace, and N Littenberg, 'Medical Education on Human Trafficking', AMA Journal of Ethics, vol. 17, issue 10, pp. 914-921, https://doi.org/10.1001/ journalofethics.2015.17.10.medu1-1510. 
Providers can then refer patients for primary care, mental health treatment, and other support, hopefully through established connections with service providers in their communities. Care for trafficked persons is most effective when it is comprehensive (addresses more than one body system) and integrated (addresses social, emotional, and legal needs). ${ }^{45}$ Therefore, the ED healthcare provider is a significant part of a team that can begin to delegate the often-complex needs of trafficked patients to appropriate experts.

\section{Incorporating Training into Healthcare Education}

Three studies reviewed here included learners, such as medical and nursing students, in their participant pool. Consistently in the literature, medical and nursing trainees report a lack of formal education on human trafficking in their curricula, with one survey of medical schools in the United Kingdom showing that 72 per cent did not provide trafficking education of any kind and 70 per cent had no plans for doing so. ${ }^{46}$ The few trainings that focused on students showed promising outcomes, including increased student confidence in interacting with trafficked patients and increased trainee interest in pursuing trafficking as a research topic. ${ }^{47}$ Diverse educational formats like simulation and case-based learning can be impactful on students' interactions with trafficked patients when they encounter them in the hospital setting. ${ }^{48}$ More research is required in the medical and nursing school education domain to determine how interventions for students and institutions can be effective on a long-term basis.

\section{Prioritising Marginalised Groups}

One study highlighted the importance of recognising patients at higher risk of being trafficked, including youth and racially or ethnically minoritised groups, and individuals who do not have work or residency permits (Cole et al.). In addition to survivor participation in education, we agree with the recommendations given by The Survivor Council from the Presidential Task Force on Human Trafficking that there should be increased funding for research and treatment for these underserved groups in the trafficking domain, including boys and men, LGBTQIA+ individuals, indigenous populations, and people with special

45 C Zimmerman, et al., The Health Risks and Consequences of Trafficking in Women and Adolescents: Findings from a European study, London School of Hygiene \& Tropical Medicine, London, 2003.

46 Arulrajah and Steele.

47 RM Lutz, 'Human Trafficking Education for Nurse Practitioners: Integration into standard curriculum', Nurse Educ Today, vol. 61, 2018, pp. 66-69, https://doi. org/10.1016/j.nedt.2017.11.015.

48 H Stoklosa, et al., 'Medical Education and Human Trafficking: Using simulation', Med Educ Online, vol. 22, issue 1, 2017, https://doi.org/10.1080/10872981.2017.1412746. 
needs or disabilities. These marginalised groups face structural discrimination that increases the risk of being trafficked. Furthermore, because they are often excluded from awareness efforts, their health outcomes may be more negatively impacted. ${ }^{49}$ We therefore advocate for future educational interventions to include informed awareness of and focus on these gaps.

\section{Limitations}

The limitations of our study include the decreased rigor of a scoping compared with a systematic review. We chose a scoping review due to our study objective, which was exploratory rather than to rigorously assess the quality of each study through a validated measure. One strength of our study is the inclusion of poster presentations, abstracts, and thesis papers from the 'grey literature', which highlight how the topic of human trafficking education for ED providers is rapidly emerging.

Overall, our review found a lack of studies, but also promising outcomes for providers and patients when training is implemented. We advocate for the development of educational interventions that are team-based (e.g., involve all ED team members and relevant community partners); include self-reported outcomes measures (e.g., pre- and post-surveys); and are patient-centred and informed by survivors. Additionally, we endorse the development of interventions that fit with the unique timing and physical constraints of the ED workflow. More research is needed to develop a validated educational tool for ED providers and compare interventions for participant and patient outcomes. Future studies might also consider investigating patient outcomes pre- and post-intervention in addition to provider perceptions.

\section{Conclusion}

Human trafficking education is a critical opportunity to increase ED staff preparedness and positively impact outcomes for trafficked patients. Though the ED may be considered a challenging place to care for trafficked patients due to the nature of this busy, fast-paced environment, our review demonstrates that, if staff are adequately trained and protocols are in place, the ED can be an ideal setting to engage these patients in care. Educational interventions need to fit into the busy environment of an ED, ideally to be completed through flexible modalities (either online or in-person) during work hours, and include as many members of clinical and non-clinical staff as possible. Education must allow

49 United States Advisory Council on Human Trafficking, Annual Report 2020, US Department of State, 2020, https://www.state.gov/united-states-advisory-councilon-human-trafficking-annual-report-2020. 
space for providers' discomfort with the topic of trafficking while recognising the ED encounter as a window of opportunity in a trafficked patient's journey. We strongly recommend that states and countries mandate trafficking trainingwith pre- and post-test results-in recertification programs for ED staff. Such trainings should be developed in conjunction with survivors because they are the experts on content, and their expertise should be valued and compensated.

\section{Acknowledgments}

The authors would like to acknowledge the contributions of Fay Chelmow, Dr David Chelmow, and John Cyrus in the drafting of this manuscript; their guidance was indispensable.

Caroline ('Carrie') Shadowen, MD, is an incoming resident in Obstetrics \& Gynaecology at the Virginia Commonwealth University Health System (VCUHS). She studied English at the University of California, Berkeley and is a strong believer in making the world safer for everyone, especially groups who have historically been ignored and mistreated. Her clinical and research interests focus on the intersection of women's health and substance use disorders. She was the Outreach Chair for the VCUHS Third Annual Human Trafficking Symposium. Email: cshadowen@gmail.com

Sarah Beaverson is a third-year medical student at the Virginia Commonwealth University School of Medicine. Before medical school she attended Duke University for a dual degree in Biology and Global Health. She is one of the Research Co-Chairs of the VCUHS Student Interest Group in Human Trafficking. She is interested in family medicine and women's reproductive health, and passionate about racial health equity and community health. Her previous research experience is in family planning utilisation among Haitian women, and multisector population health improvement initiatives. Email: beaversons@vcu.edu

Fidelma B Rigby, MD, is an Associate Professor of OB/GYN in the Maternal Fetal Division of the Virginia Commonwealth University School of Medicine and Clerkship Director for the VCUHS OB/GYN department. She is Faculty Co-Leader of the VCUHS Student Interest group in Human Trafficking and has given multiple presentation on human trafficking in the community and at national OB/GYN conferences. She has been Co-Chair of the Annual VCUHS Educational Symposium on Human Trafficking for the past three years. Her other research interests include perinatal mood disorders and undergraduate medical education. Email: fidelma.rigby@vcuhealth.org 